\title{
Collision Avoidance and Aircraft
}

\author{
Separation \\ from E. J. Dickie \\ (Ministry of Transport and Civil Aviation)
}

Ir has seemed to me for a long time now that there is a real need to review objectively and dispassionately the Rules of the Air for avoiding collision. For example, it seems quite wrong to place responsibility for taking avoiding action on one of two pilots and expect the other to maintain a steady course. Such a rule assumes that each pilot is aware of the other's presence and this is, of course, by no means the case in all circumstances. Frequently it is the pilot who, under the rules, has right of way who first becomes aware of the potential danger and there may be only seconds to spare in which to take avoiding action.

This problem has been engaging our attention in the M.T.C.A. for some time along with the parallel problem of rules to be applied by ground radar controllers and we have established certain principles which may be of some interest on the marine as well as the air side:

(a) Whatever his position relative to it, the pilot of an aircraft should always have positive action to take on sighting another aircraft.

(b) Such action should be complementary to the action laid down for the other pilot but should also be sufficient in itself to prevent collision.

(c) The same principles should be applied in the case of radar controllers with the added proviso that action initiated by a radar controller on the ground must be either the same as, or complementary to, that laid down for the pilot in the same circumstances.

Mr. Fraser $(11,34)$ explained why conventional A.T.C. methods in such areas as the North Atlantic can prove very restrictive and wasteful of airspace and we also read how difficult it is to provide safety solely by means of airborne devices. I suggest that the solution is a combination of the two methods. It lies in appreciating both the qualities and limitations of ground based A.T.C. and 'self-help' airborne devices, using the former to reduce the probability of close proximity to a very low level and the latter to detect-by direct measurementnot instances where separation has already become undesirably low, or instances where separation is less than that planned by A.T.C. but instances where separation is liable to become undesirably low for safety if no action is taken.

It is now fairly generally known that as things stand today the North Atlantic controller has to assume that if separation threatens to reduce below some 120 miles or more then the position is unsafe. This may be so on a very few occasions -my own guess is about once in ten years-but quite obviously it is not so on the vast majority.

Safety in the air can only be had at a price. If reliance continues to be placed solely on conventional methods of navigation and air traffic control the price will be increasing delays as traffic builds up. The alternative is to face up to the cost of much improved airborne navigation, communication and other equipment such as the relative position indicator referred to by Mr. Butler (I I, 65). There is, I feel sure, tremendous scope for an A.T.C. system which combines 
ground control, air navigation and air-to-air interrogation in the right proportions, the main function of the last mentioned being not to give the pilot lastminute warning of a pending disaster but to confirm to him all the time that sufficient separation for safety does in fact exist between his own and the nearest aircraft at his own flight level.

\section{from V. H. King \\ (Decca Navigator Company)}

IN areas of low traffic density it has been suggested, proximity warning indicators (or relative position indicators) could partially or entirely take over the present function of air traffic control in the sense that they may be used by pilots to determine the need to alter altitude and/or heading in order to secure safe separation from other aircraft and, at the same time, ensure the ability of aircraft to utilize optimum flight paths and altitudes with the minimum of restriction and delay. The North Atlantic has been mentioned as a typical area where this could be done.

The air traffic control system is not perfect and much has to be done to develop it to the stage when it can adequately cope with the present and future air traffic situations. The means to do this are now available in the form of high accuracy area coverage navigational aids and ground radar, and are becoming available in the form of improved data transfer systems, computers and ground presentation displays now in the course of development. Other refinements and improvements are also within reach. To discard the air traffic control system at this stage, or to relegate it to a passive role in the collision avoidance question in favour of a promiscuous form of individual pilot traffic control on the basis of airborne proximity warning indicators-which may have inherent limitations anyway-is not only a backward step but savours of a 'return to the jungle' approach.

The trend should rather be towards the improvement and extension of positive ground control to eliminate the possibility of collisions altogether, if possible. The traffic pattern should, therefore, be disciplined and orderly in the overriding interests of overall safety and appropriate to vehicles capable of closing speeds of up to 1000 m.p.h. Ideally, air traffic control should be exercised by unified civil/military centres to ensure that both main types of traffic are subjected to the same control. Given the tools, air traffic control can do the job which, after all, it was created to do. The part to be played by airborne proximity warning indicators-and, later, by developed airborne collisionavoidance systems - will then be, as it should be-not as a form of traffic control, but as a separation monitor and as a final safety factor to provide against human error.

\section{Visual Avoidance in the Air}

$$
\text { from Dr. Hentschel }
$$

By far the majority of air collisions occur in the vicinity of airports, in full daylight, and in clear weather; and in the majority of cases, distant planes are affected. 